\title{
Zum Verhältnis von Zinssatz und Wachstumsrate: Theorie und empirische Evidenz
}

\author{
Wir setzen uns in diesem Beitrag mit einigen kritischen Kommentaren von Hans-Werner \\ Sinn, Peter Bofinger und Eckhard Hein auseinander, die diese zu unserem Buch „Sparen \\ und Investieren im 21. Jahrhundert - Die große Divergenz" im Augustheft 2020 des \\ Wirtschaftsdienst abgegeben haben. Wir befassen uns insbesondere mit dem umstrittenen \\ Phänomen der dynamischen Ineffizienz, mit der Kritik an unserem Konzept der impliziten \\ Staatsverschuldung, mit der Interpretation von ökonomischen Weltbildern sowie dem Vorwurf, \\ monetäre Aspekte in unserer Theorie vernachlässigt zu haben.
}

\begin{abstract}
Mit dem Buch „Sparen und Investieren im 21. Jahrhundert Die große Divergenz" haben wir eine umfassende Erklärung sowie empirische Evidenz für das weltweit zu beobachtende Phänomen der Niedrigzinsen vorgelegt und verschiedene darauf basierende wirtschaftspolitische Handlungsempfehlungen abgegeben. Unsere Untersuchung ist auf große Resonanz gestoßen. Im Folgenden wollen wir uns mit einigen kritischen Kommentaren von Hans-Werner Sinn, Peter Bofinger und Eckhard Hein auseinandersetzen, die im Zeitgespräch im Augustheft 2020 des Wirtschaftsdienst dokumentiert sind.
\end{abstract}

Neue Denkansätze treffen zunächst bei vielen Kommentatoren auf Unverständnis und auf Widerspruch. Auch diejenigen Ideen, die letztlich allgemein anerkannt werden, mögen zunächst noch nicht ausreichend erklärt oder in Gänze verstanden werden; manchmal ist auch ihre Zeit einfach noch nicht gekommen. Hinzu kommt, dass das Verharren in eingespielten Denkmustern eine ausgeprägte individuelle und auch Forschungsgemeinschaften (im Sinne von Thomas Kuhn)

Prof. Dr. Carl Christian von Weizsäcker ist emeritierter Professor für Volkswirtschaftslehre der Universität zu Köln und Senior Research Fellow des MaxPlanck-Instituts zur Erforschung von Gemeinschaftsgütern in Bonn.

Prof. Dr. Hagen Krämer ist Professor für Volkswirtschaftslehre an der Fakultät für Wirtschaftswissenschaften der Hochschule Karlsruhe - Technik und Wirtschaft. prägende Eigenschaft ist. Die Ideengeschichte der Wirtschaftswissenschaften liefert hierfür zahlreiche Anschauungsbeispiele. Hierzu ein Zitat von John Maynard Keynes, das wir an den Anfang unseres Buches gesetzt haben: „The difficulty lies, not in the new ideas, but in escaping from the old ones, which ramify, for those brought up as most of us have been, into every corner of our minds." (Keynes 1936, viii)

Risiko, Sachkapitalinvestitionen und dynamische Ineffizienz

Eine für die weltweite Debatte über das Thema Staatsschulden zentrale Frage ist die nach der „dynamischen Ineffizienz". Kann die volkswirtschaftliche Grenzproduktivität des Kapitals und damit inr Preissignal in der Form der Kapitalrendite dauerhaft unter der volkswirtschaftlichen Wachstumsrate liegen? Der zentrale Vorwurf, den Hans-Werner Sinn gegenüber unserem Ansatz vorbringt, lautet, dass unter den von uns beschriebenen Voraussetzungen dynamische Ineffizienz auftreten müsse (Sinn, 2020, 573). Und dies könne nicht sein, da dynamische Ineffizienz in einer freien Marktwirtschaft per se nicht auftreten könne. Wir vertreten dagegen die Ansicht, dass heutzutage Bedingungen existieren, die dazu führen können, dass der Zins dauerhaft unter der Wachstumsrate bleibt und dynamische Ineffizienz hervorruft.

Welche „Kapitalrendite“ ist das korrekte Preissignal für die volkswirtschaftliche Grenzproduktivität des Kapitals? Völlig zu Recht verweist Sinn darauf, dass unsere Antwort und die von Piketty sich gegenseitig ausschließen. Unsere Antwort

(C) Der/die Autor(en) 2020. Open Access: Dieser Artikel wird unter der Creative Commons Namensnennung 4.0 International Lizenz (https:// creativecommons.org/licenses/by/4.0/deed.de) veröffentlicht.

Open Access wird durch die ZBW - Leibniz-Informationszentrum Wirtschaft gefördert. 
ist, dass dies der risikobereinigte und inflationsbereinigte Zinssatz $r$ ist. Daraus folgt heutzutage die Diagnose dynamischer Ineffizienz, da dieser Zins seit längerem unter der volkswirtschaftlichen Wachstumsrate $g$ liegt. Piketty bestimmt die Kapitalrendite ganz anders als wir. Seine Kapitalrendite ist höher als die Wachstumsrate. Das führt nach seiner Theorie zusammen mit einer hohen Spar- bzw. Investitionsquote der Kapitalbesitzer zu einer ständigen Erhöhung des Anteils der Kapitalerträge am Volkseinkommen. Die Piketty-These impliziert damit den Weltzustand dynamischer Effizienz.

\section{Risikofreier Zins und Risikoprämie}

Wie auch Sinn feststellt, ist für die richtige Antwort ganz entscheidend der Zusammenhang zwischen der Risikoprämie und der Rendite auf Sachkapital. Er verweist in diesem Zusammenhang auf seine Antrittsvorlesung an der Universität München. Als eine Möglichkeit sieht er die Hypothese, dass es einen strikt komplementären Zusammenhang zwischen Risiko und Sachkapitalbestand gibt. Diese Hypothese impliziert, dass man aus der Risikoprämie einen einfachen Zuschlag zur Kapitalrendite macht. Letztere wird damit ohne Zweifel höher als die Wachstumsrate. Damit wären wir nahe am Piketty-Ansatz, der zwar von Sinn in anderen Publikationen heftig kritisiert wird, den er aber in seinem jetzigen Beitrag implizit übernimmt: er weist auf das Faktum hin, dass die Profitquote höher als die Investitionsquote ist. Dies sei ein Gegenbeweis unserer These, aus der folge, dass die Investitionsquote höher als die Quote der Kapitalerträge sein müsse. Damit hat Sinn die Risikoprämie voll den Kapitalerträgen zugerechnet. Diese Zurechnung ist irrtümlich - und insofern sind sowohl Sinn als auch Piketty mit ihrer Analyse auf dem Holzwege. Wer Risiko übernimmt, investiert ja nicht nur in Sachkapital, sondern ist als Unternehmer auch mit zahlreichen anderen, komplementären Produktionsfaktoren befasst. Sachkapital ohne andere Produktionsfaktoren, wie z.B. Arbeit oder Boden, ist völlig nutzlos.

Wir schlagen vor, den Zusammenhang zwischen Risiko und Sachkapitalinvestitionen durch folgende Kategorisierung von Sachkapitalinvestitionen aufzuschlüsseln: Sie können das Gesamtrisiko, das ein Unternehmer trägt, erhöhen. Dann sprechen wir von offensiven Sachkapitalinvestitionen. Sie können aber auch das Gesamtrisiko, das der Unternehmer trägt, vermindern. Dann sprechen wir von defensiven Sachkapitalinvestitionen. Und Sachkapitalinvestitionen können dazwischen liegen, also keinen merklichen Einfluss auf das Gesamtrisiko haben, das der Unternehmer trägt. Dann sprechen wir von neutralen Sachkapitalinvestitionen. Den offensiven ordnen wir eine positive Risikoprämie zu, den defensiven eine negative Risikoprämie, und den neutralen eine Risikoprämie von Null. Eine negative Risikoprämie bedeutet, dass man die Investition selbst dann durchführt, wenn der Erwartungswert ihrer Rendite unter den Finanzierungskosten liegt. Man denke z. B. an eine Einbruchs-Alarmanlage, die das Vermögensrisiko durch Einbrüche vermindert. Die bisherige Makroökonomie ist davon geprägt, dass man einen engen positiven Zusammenhang zwischen dem unternehmerischen Risiko und der Verwendung von Sachkapital unterstellt. Wir kommen aufgrund mikroökonomischer Überlegungen zu anderen Ergebnissen. Hierzu einige Beispiele:

\section{Risikoprämie auf das Gesamtgeschäft}

Erstes Beispiel: Was ist das Hauptrisiko eines Geschäftsmanns? Es ist die Gefahr, dass seine Kunden zu einem Konkurrenten abwandern. Diesem Risiko steht die Chance gegenüber, dass die Kunden des Konkurrenten zu ihm kommen. Nur wenige Menschen würden sich scheuen unternehmerisch tätig zu werden, wenn sie sich ihrer Kunden bei einem Preis oberhalb ihrer Durchschnittskosten sicher sein könnten. Aber nur wenige Menschen wagen sich in die Selbständigkeit bei intensivem Wettbewerb, wenn sie sich auch für einen gut bezahlten Job als Arbeitnehmer entscheiden können.

Es bedarf daher einer Risikoprämie für das Engagement als Geschäftsinhaber. Ohne sie gäbe es kein Gleichgewicht zwischen Angebot und Nachfrage bei unternehmerischen Leistungen (Nachfrage hier verstanden als der volkswirtschaftliche Bedarf bei Vollbeschäftigung). Der Wettbewerb ist der Zwang zur betrieblichen Rationalisierung, zur Disziplinierung des eigenen Lebens, zum Aufbau und zur Aufrechterhaltung des eigenen Humankapitals, das aus Fachwissen, Zielorientierung, Durchsetzungsfähigkeit, Kundenfreundlichkeit, Reputation (Goodwill) in der eigenen geschäftlichen Umgebung besteht. Es ist im Grunde dieses Humankapital, das mit dem Geschäftsrisiko belastet ist. Denn es muss da sein, bevor man rationell in Sachkapital investieren kann. Die Zurechnung der Risikoprämie zum Sachkapital ist damit grundverkehrt. Sachkapital dient dem Geschäftsmann primär zur Absicherung des überwiegend schon investierten Humankapitals. Wenn sich ein Angestellter selbständig macht, dann hat dieser Neu-Unternehmer sein Humankapital schon weitgehend gebildet, ehe er Unternehmer wird. Man denke als Beispiel an eine in einer Klinik angestellte Ärztin, die nun eine eigene Praxis aufmacht. Sie bringt ihr Humankapital schon mit - neben ihrer jahrelangen Ausbildung nicht zuletzt die Reputation, die sie sich dadurch erworben hat, in einer angesehenen Klinik jahrelang gearbeitet und gelernt zu haben.

Die Investition in Sachkapital ermöglicht zusammen mit den anderen Produktionsfaktoren die Leistung, die die Kundschaft beim Geschäftsinhaber nachfragt. Wie weit geht der Unternehmer bei diesen Sachinvestitionen? Im Wettbewerb geht es inm unter anderem um seine stete Lieferfähigkeit. In 
der Regel verkauft er ja zu einem Preis oberhalb der Grenzkosten. Er ist somit ceteris paribus fast immer daran interessiert, mehr zu verkaufen als er tatsächlich Absatz hat. Daher macht praktisch jeder Geschäftsinhaber Werbung. Außerdem hängt seine gute Reputation beim Kunden an seiner jederzeitigen Lieferfähigkeit. Der Unternehmer sorgt durch seine Sachinvestition dafür, dass seine Lieferkapazitäten ein ganzes Stück größer sind als sein durchschnittlicher Absatz, also größer als der Erwartungswert seines Absatzes. In einem Wettbewerbsmarkt (bei der vorherrschenden heterogenen Konkurrenz à la „monopolistic competition“) gibt es Reservekapazitäten. Diese machen intensiven Wettbewerb erst möglich; aber sie sind in der Regel höher als sie bei einem hypothetischen gemeinnützigen Monopolisten wären, der denselben Markt bedienen würde und der, prozentual gerechnet, eine viel geringere Schwankung der Nachfrage im Auge haben muss.

Daraus folgt, dass Sachkapital in diesem Markt bis zu einem Punkt investiert wird, wo die volkswirtschaftliche Rendite geringer ist als die betriebliche im Sinne einer hohen Lieferfähigkeit. Die betriebliche Rendite entspricht aber einer neutralen Investition, da das Geschäftsrisiko schon von dem vorgängig investierten Humankapital in Anschlag gekommen ist. Sofern der Unternehmer das Sachkapital aus eigenem Vermögen finanzieren kann, entspricht damit dessen erwartete Rendite der risikobereinigten Rendite dieses Vermögens, sprich, heutzutage, der Rendite von risikofreien Staatsanleihen. In diesem Fall haben wir im Durchschnitt der Fälle eine Sachkapitalrendite vor uns, die nicht höher ist als die von risikofreien Staatsanleihen, wohl aber höher als die volkswirtschaftliche Grenzproduktivität des Kapitals.

Wichtig ist der Gedanke, dass eine Risikoprämie auf das Gesamtgeschäft bezogen ist - und nicht primär auf die damit zusammenhängenden Sachkapitalinvestitionen. Die Entscheidung, selbständig zu werden, wird unter Abwägung der damit zusammenhängenden Chancen und Risiken getroffen. Dort ist die erwartete Risikoprämie entscheidungsrelevant. Würde man die erwartete Risikoprämie dann dem später zu investierenden Sachkapital erneut zurechnen, so wäre das eine Doppelzählung. Sie wäre ein Denkfehler.

\section{Immobilien und Risikoprämie}

Zweites Beispiel: Eine analoge Betrachtung können wir bei Immobilieninvestitionen zu Renditezwecken anstellen. Wenn jemand ein Wohnhaus zwecks Vermietung z.B. in München baut oder kauft, dann ist ein erheblicher Teil des Kaufpreises oder der Gesamterstellungskosten dem Wert des Bodens zuzurechnen, der das Baugrundstück bildet. Dieser Bodenwert errechnet sich aus der künftigen Bodenrente, abgezinst auf die Gegenwart. Wenn der Investor das noch leerstehende Grundstück kauft, so ist er bereit, einen Preis zu zahlen, der sich aus dem Erwartungswert des künftigen Überschusses der jährlichen Nettomieteinnahmen über die Verzinsungs- und Abschreibungskosten des zu errichtenden Gebäudes ableitet. Dabei kommt ein Diskontierungsfaktor ins Spiel, in dem neben dem risikofreien Zinssatz eine Risikoprämie enthalten ist. Der Risikoabschlag für die Bewertung des Gesamtprojekts ist also schon in dem Kaufpreis des noch unbebauten Grundstücks enthalten. Es wäre nun ein Rechenfehler, wenn man bei den Verzinsungskosten der Gebäudeinvestition die Risikoprämie erneut in Anschlag bringen würde. Dieser Rechenfehler würde im Übrigen damit bestraft, dass man das leere Bauland gar nicht kaufen könnte, weil ein Bieter, der diesen Rechenfehler vermeidet, mehr dafür bezahlen und damit den Zuschlag erhalten würde. Für einen Käufer und Bauherren, der die Gesamterstellungskosten des Wohnhauses selbst aufbringt, wäre damit der Zinssatz für die Zinskosten der Bauaufwendungen der risikofreie Zinssatz.

Das folgende Zahlenbeispiel mag das Verständnis für diesen Sachverhalt erhöhen. Der potenzielle Käufer einer Bauparzelle sieht im Erwartungswert eine konstante Netto-Jahresmiete für das bebaute Grundstück von 40.000 Euro voraus. Die Baukosten veranschlagt er mit 550.000 Euro. Der risikofreie Zinssatz sei $1 \%$ p.a. Der potenzielle Käufer will eine Risikoprämie von $3 \%$ p.a. erreichen. Damit diskontiert er die künftigen Mieteinnahmen mit einer Rate von $4 \%$ p.a. $=$ Risikoprämie plus risikofreier Zinssatz. Daraus ergibt sich ein subjektiver Wert des Gesamtprojekts von 1 Mio. Euro. Damit ist er bereit, für die unbebaute Landparzelle bis zu 450.000 Euro zu bieten, sodass inn das Gesamtprojekt maximal 1 Mio. Euro kosten würde. Will der Käufer eine Risikoprämie von $4 \%$ p.a. erreichen, so diskontiert er den Erwartungswert des künftigen Stroms von Mieteinnahmen mit $5 \%$ p.a. Hieraus ergibt sich ein subjektiver Wert des Gesamtprojekts von 800.000 Euro. Er ist dann bereit, bis zu 250.000 Euro für die unbebaute Parzelle zu bieten.

\section{Dominanz risikomindernder Sachkapitalinvestitionen}

Drittes Beispiel: Natürlich kann man Beispiele offensiver Investitionen finden. Indessen scheinen sie uns in der Minderheit zu sein. Denn immer dann, wenn es eine relativ enge Komplementarität zwischen anderen Investitionen und den Realkapitalinvestitionen gibt, spricht eine hohe Wahrscheinlichkeit dafür, dass die komplementären Investitionen zeitlich vorgängig sind und daher das Gesamtrisiko absorbieren. Wenn z.B. eine schon seit langem im Geschäft etablierte Unternehmung über eine gut eintrainierte Vertriebsmannschaft verfügt, dann darf diese nicht deshalb im Verkaufen gebremst sein, weil es an der Produktionsfähigkeit wegen zu geringer Sachinvestitionen hapert. Eine korrekte Rentabilitätskalkulation wird immer dazu führen, dass die Sachinvestitionen in Produktionsanlagen und Logistik nicht der 
Engpass für den Absatz sind. Auch hier wird der Tendenz nach gelten, dass diese quasi nachlaufenden Sachinvestitionen das Gesamtrisiko des Geschäfts eher vermindern als anheben.

\section{Defensive Sachinvestitionen überwiegen}

Viertes Beispiel: Wir vermuten, dass die neutralen und defensiven Investitionen in Sachvermögen die offensiven Investitionen wertmäßig überwiegen. Dies zumal, weil es auch eine Fülle defensiver Investitionen in Sachvermögen gibt, die gar nicht als „Investitionen“ verbucht werden. Ein Beispiel ist der Aufwand für Instandhaltung. Dieser wird fast immer den laufenden Betriebskosten zugerechnet. Das ist insofern nicht völlig korrekt, als der laufende Betrieb über die kurze Frist auch mit wesentlich weniger Instandhaltungsaufwand weitergeführt werden kann. Denn wie wäre es sonst möglich, dass Unternehmen gelegentlich der Vorwurf gemacht wird, dass sie ihre Gewinne dadurch „verschönern“, dass sie an Instandhaltungsaufwand übermäßig sparen. Man denke an die Deutsche Bahn AG, für die ein Börsengang geplant war. Um einen guten Gewinnausweis zu bringen, wurde an Instandhaltungsaufwand übermäßig gespart. Der langfristig optimale Instandhaltungsaufwand dient dazu, das Risiko künftiger Betriebsunterbrechung auf ein optimales Maß zurückzuführen, wobei sich dieses beeinflussbare Risiko erfahrungsgemäß auf mehrere Jahre verteilt. Insofern ist Instandhaltungsaufwand eine Investition - und zwar eine das betriebliche Gesamtrisiko vermindernde, also eine defensive Investition.

Vielfach werden Investitionen durch Kreditaufnahme fremdfinanziert. Handelt es sich dabei um neutrale Investitionen, wird die Investition bis zu dem Punkt ausgedehnt, wo die erwartete marginale Ertragsrate dem Kreditzinssatz entspricht, der an die Bank gezahlt werden muss. Wir haben in von Weizsäcker und Krämer (2019) in Kapitel 2.2 ausführlich dargelegt, weshalb auch dann die volkswirtschaftliche Rendite dem risikofreien Zinssatz für Staatsanleihen entspricht. Kurz zusammengefasst liegt der Grund darin, dass man den Ressourcenaufwand der Bank in Abhängigkeit ihres Kreditgeschäfts mitberücksichtigen muss. Wir kommen damit zu der Vermutung, dass Sachkapital im Durchschnitt der Volkswirtschaft keineswegs mit einer positiven Risikoprämie verbunden ist. Natürlich gibt es in einer gut funktionierenden Marktwirtschaft unter der Bedingung der Vollbeschäftigung im Durchschnitt erhebliche Einkommensanteile, die als Risikoprämie angesehen werden können. Sie aber grundsätzlich auf das eingesetzte Sachkapital zu beziehen und daraus eine ausgeweitete Kapitalrendite zu konstruieren, ist verfehlt. Dass große Teile der Einkommen aus Risikoprämien den höheren Einkommensschichten zufallen, bewirkt eine hohe Sparquote aus Risikoprämien. Diese Ersparnisse tragen damit zu einer Senkung und nicht zu einer Steigerung des Zinssatzes bei. Die hohen Bestände an Staatsanleihen bei den besonders erfolgreichen Unternehmen der digitalen Welt sind ein sinnfälliges Beispiel für diese allgemeine Aussage.

\section{Enteignungsgefahr bei Boden und Sachkapital}

Sinn verteidigt weiterhin die Niehans-Homburg-Theorie, dass wegen des Produktionsfaktors Boden der Gleichgewichtszins gar nicht unter die Wachstumsrate fallen könne. Er wehrt unseren Hinweis auf die Enteignungsgefahr von Bodenrenten mit dem Argument ab, dass doch im linken Lager (er nennt speziell den Juso-Vorsitzenden Kevin Kühnert), mindestens so stark für eine Enteignung von Realkapital plädiert wird. Dieses Argument ist nicht stichhaltig. Wir weisen in unserem Buch darauf hin, dass empirisch $90 \%$ des Bodenwerts in der Region OECD plus China aus städtischem Boden besteht, der entweder bebaut oder Bauland ist. Hier gelten andere ökonomische Gesetze als beim Sachkapital. Droht dem Sachkapital Enteignung oder erhöhte Besteuerung, dann führt dies zu einem Rückgang der Sachinvestitionen mit der Folge, dass die Rendite auf Sachvermögen solange steigen muss, bis der Investitionsanreiz ebenso hoch ist wie vor der Enteignung oder Besteuerung. Die Besteuerung von Sachkapital kann letztlich voll überwälzt werden. Dieser Überwälzungsprozess in der Form extremer Investitionszurückhaltung ist ein gesamtwirtschaftlich höchst schmerzvoller Prozess. Das würde selbst ein hypothetischer Wirtschafts- oder Finanzminister Kevin Kühnert verstehen und deshalb vor solchen Enteignungsversuchen zurückschrecken, schon um die nächste Wahl zu gewinnen.

Ganz anders sieht das bei Bodenrenten aus städtischem Boden aus. Werden diese besteuert oder durch staatliche Regulierung (etwa durch Mietrecht) teilenteignet, so können sich die Bodenbesitzer dagegen nicht durch Anhebung der Bodenrenten schadlos halten. Die Überwälzbarkeit ist bei freier Preisbildung auf dem Markt für Immobilienmieten nicht gegeben. Die Bruttobodenrente bleibt von der Bodensteuer in erster Approximation unberührt und wird durch die Bodensteuer nur anders verteilt. Im Übrigen gibt es gute allokationspolitische Gründe dafür, die Bodenrente aus städtischem Boden fiskalisch zu belasten - zugunsten einer Absenkung der allokationsverzerrenden Einkommens- und Umsatzsteuern (z.B. Arnott und Stiglitz, 1979; von Weizsäcker und Krämer, 2019, Kapitel 5).

In die Irre führt auch das Argument von Sinn, dass man erst zum Zeitpunkt „Unendlich“ feststellen kann, ob dynamische Ineffizienz vorliegt oder nicht. Natürlich kann man Modelle konstruieren, wie Sinn es vor einigen Jahren getan hat, in denen von einem sehr niedrigen Niveau aus die volkswirtschaftliche und privatwirtschaftliche Kapitalrendite allmählich auf ein Niveau oberhalb der Wachstumsrate ansteigt. 
Genauso gut kann man Modelle konstruieren, in denen es von einem Zustand hoher Renditen allmählich zu einem dauerhaften Realzins unterhalb der Wachstumsrate kommt. Die Zukunft ist ungewiss. Als pragmatischer Indikator für die beste Staatsschuldenpolitik eignen sich nur die gegenwärtige volkswirtschaftliche Kapitalrendite und ihre wahrscheinliche Entwicklung in der näheren Zukunft. Die Gefahr für den Euro und für das weltwirtschaftlich produktive Regime des freien internationalen Handels ist spätestens seit der USPräsidentschaftswahl vom 8. November 2016 offensichtlich; aber sie wird massiv verstärkt, wie wir in unserem Buch darlegen, durch die deutsche Schuldenbremse mit den sie begleitenden Leistungsbilanzüberschüssen Deutschlands. Hier spielt der Realzinssatz in hundert oder zweihundert Jahren keine Rolle.

\section{Implizite Staatsschulden}

Einen wichtigen Punkt in unserer Argumentation stellt die Behandlung von Ansprüchen dar, die die Beitragszahler durch ihre Einzahlungen in die sozialen Versicherungssysteme erworben haben. Für uns stellen diese Anwartschaften Vermögensansprüche der privaten Beitragszahler dar, während sie auf der anderen Seite als Staatsschulden angesehen werden müssen. Da sie andersartige Verbindlichkeiten sind als staatliche Wertpapiere, bezeichnen wir sie als implizite Staatsschulden, während die herkömmlichen Staatsschulden explizite Staatsschulden darstellen. Wir argumentieren, dass sowohl die expliziten als auch die impliziten Staatsschulden für die Bürger Vermögen darstellen. Die impliziten Staatsschulden sind vom Umfang her sehr bedeutsam. Nach unseren Berechnungen besteht in der Region OECD plus China ungefähr ein Drittel des privaten Vermögens aus impliziten Staatsschulden.

Peter Bofinger vertritt dagegen die Auffassung, dass bei „... dieser Betrachtungsweise zwar die aus dem Umlagesystem vom Staat an die Privaten zu leistenden Zahlungen berücksichtigt [werden]. Die gleichzeitig von den Privaten an den Staat zu leistenden Beiträge für die Rentenversicherung [bei von Weizsäcker und Krämer] jedoch ausgeblendet werden. Wenn man implizite Staatsschulden ausweist, sollte man auch die impliziten Staatsforderungen berücksichtigen" (Bofinger, 2020, 580). Bofinger meint daher, dass sich die Barwerte der Zahlungsströme von impliziten Staatsforderungen und zu leistenden Zahlungen der privaten Haushalte entsprechen würden. Davon leitet er die Behauptung ab, dass der Privatsektor keine Netto-Forderungen gegenüber dem Staat haben würde. Gegen diese Sichtweise lassen sich einige Gegenargumente vorbringen. Wer wie Bofinger diese Ansicht vertritt, müsste konsequenterweise auch den expliziten Staatsschulden die zukünftigen Steuereinnahmen gegenüberstellen. Ein derartiges Verrechnen von bereits existierenden Verbindlichkeiten und für die Zukunft erwarteten
Zahlungseingängen wird allerdings nicht nur in den Volkswirtschaftlichen Gesamtrechnungen aus guten Gründen unterlassen. Auch die in der Privatwirtschaft üblichen Bilanzierungsvorschriften verlangen, dass nur die gegenwärtigen Verbindlichkeiten, nicht jedoch zukünftig erwartete Einnahmen berücksichtigt werden. Künftige Zahlungsverpflichtungen, die ein Unternehmen beispielsweise aufgrund von zugesagten Betriebsrenten hat, müssen in der Bilanz als Pensionsrückstellungen passiviert werden. Umgekehrt dürfen die erwarteten Einnahmen, selbst wenn sie dem Unternehmen von Dritten vertraglich zugesichert wurden (z.B. im Rahmen langfristiger Lieferverträge), nicht aktiviert werden, so wie dies der Energiekonzern Enron um die Jahrtausendwende zur „Verschönerung“ seiner Bilanz und seiner Gewinn- und Verlustrechnung dennoch getan hat.

Dieser Idee folgt auch die neu gestaltete Vermögensrechnung in den Volkswirtschaftlichen Gesamtrechnungen, wie sie bereits im System of National Accounts (SNA) 2008 bzw. im Europäischen System Volkswirtschaftlicher Gesamtrechnungen (ESVG) 2010 neu eingeführt worden ist. Im Rahmen eines Satellitensystems (konkret: in einer Ergänzungstabelle zum Kernsystems der VGR) werden die kapitalisierten Ansprüche aus allen arbeitgeberfinanzierten Alterssicherungssystemen, darunter auch die Rentenansprüche gegenüber der gesetzlichen Rentenversicherung dargestellt, die durch eine aktuarische Kalkulation nach international einheitlichen Vorschriften ermittelt werden. Die OECD erkennt die Bedeutung dieser Ausweitung der Informationsbereitstellung für die Staatsverschuldung an: „This extension could have a major impact on the measurement of the government debt, because unfunded retirement plans [...] involve heavy liabilities in some countries." (Lequiller und Blades, 2014, 447)

Die Mitgliedstaaten der EU sind verpflichtet, alle drei Jahre die „im Rahmen von Sozialschutzsystemen aufgelaufenen Alterssicherungsansprüche" zu berechnen und diese an das Statistische Amt der Europäischen Union (Eurostat) zu übermitteln (Haug, 2018, 78). Zur Berechnung des Kapitalwerts der Verbindlichkeiten des Staates aus den Alterssicherungssystemen findet dabei das Konzept der „Accrued to Date Liabilities“ Anwendung. Hierbei werden nur die in der Vergangenheit von den heutigen Rentnern oder Beitragszahlern erworbenen Ansprüche einbezogen. Ansprüche, die nach einem definierten Stichtag erworben wurden oder die in der Zukunft noch erworben werden könnten, werden bei dieser Methode nicht berücksichtigt. Ermittelt wird dadurch der Barwert der Anwartschaften, die existieren, wenn die Rentenversicherung zum Bilanzstichtag schließen würde. Die Sozialversicherung ist mit ihrem Umlaufverfahren daher letztlich wie eine private Lebensversicherung konzipiert - jedoch ohne den Deckungsstock. Die sozialstaatlichen Verpflichtungen des Staates sind deshalb als impliziter Teil der Staatsschulden zu behandeln. 
Bofinger hat grundsätzlich Recht mit seiner Feststellung, dass die Anwartschaften der Versicherten gegenüber der Sozialversicherung der Höhe nach nicht in vollem Umfang rechtlich garantiert seien. Schließlich kann der Gesetzgeber den Leistungsumfang der Sozialversicherung prinzipiell verändern. Aber auch dieser Einwand kann letztlich nicht überzeugen. Zum einen hat das Bundesverfassungsgericht die Anwartschaften aus der Sozialversicherung unter den Eigentumsschutz des Artikels 14 des Grundgesetzes gestellt und damit hohe rechtliche Hürden für einschneidende Kürzungen aufgestellt. Zum anderen unterliegt schließlich jeder Vermögenswert dem Risiko, dass entweder durch individuelle Zahlungsprobleme des Schuldners oder durch allgemeine äußere Einflüsse, wie z.B. die generelle wirtschaftliche Lage, ein Wertverlust eintreten kann. Natürlich kann man die Höhe der Anwartschaften in den Sozialversicherungen allenfalls als eine Momentaufnahme auf Grundlage der zum Bilanzierungszeitpunkt geltenden gesetzlichen Regelungen ansehen. Aber schließlich wird auch der diskontierte Gegenwartswert von zukünftig erwarteten Einnahmeströmen aus Kapitalanlagen jeglicher Form immer auf Basis der zum jeweiligen Zeitpunkt verfügbaren Informationen ermittelt. In allen Fällen gilt: die Zukunft ist ungewiss. Dass sich die Chance auf einen Werterhalt der Anwartschaften erhöht, indem die Politik dafür sorgt, dass sich die „Leistungsfähigkeit der Wirtschaft verbessert" (Bofinger, 2020, 580) trifft schließlich ganz allgemein sowohl für Sicherung des zuküntigen Wohlstands der Rentner als auch für den Werterhalt aller anderen Vermögensanlagen zu.

Zusammenfassend kann man zu diesem Punkt festhalten, dass Vermögensansprüche, die auf erwarteten Rentenzahlungen beruhen, „keineswegs unsicherer und ökonomisch weniger wert [sind] als erwartete Zinszahlungen aus Wertpapierbesitz oder verbriefte Leistungen aus privaten Versicherungsverträgen" (Spahn, im Erscheinen, 8). Anwartschaften aus der Rentenversicherung sind sicherlich eine ganz spezielle Vermögenskategorie. Sie stellen für den Bürger jedoch ebenso Vermögen dar, wie solches, das auf den herkömmlichen Vermögensmärkten gebildet wird. Das Gegenstück zu Vermögen sind Schulden - und die Schulden aus der Sozialversicherung sind für unsere Erkenntniszwecke als implizite Verschuldung im Sektor Staat zu verbuchen.

\section{Konkurrierende Weltbilder}

Peter Bofinger behauptet, dass unser Denkmodell trotz gegenteiliger Äußerungen letztlich ganz von der „klassischen Weltsicht" (Bofinger, 2020, 577) geprägt sei. Wir würden von Vollbeschäftigung ausgehen und eine Modellwelt ohne Geld zugrunde legen. Bofinger fällt mit dieser Kritik an unserem Buch allerdings weit hinter die „neoklassische Synthese" zurück, die von der Keynes nachfolgenden Generation entwickelt wurde. Sie revolutionierte mit Samuelsons (1948) einführendem Lehrbuch „Economics“ das makroökonomische Denken in Lehre und Forschung. Sie war der Weg, auf dem die "General Theory" von Keynes (1936) ihren breiten Einfluss gewann. Bei Bofinger jedoch gibt es nur ein „Entweder-Oder". Also entweder Neoklassik oder Keynes. Und für inn letztlich nur Keynes. Dieses „Entweder - Oder“ wird nicht dadurch besser, dass man es mit dem Weltbild von Kopernikus oder Ptolemäus vergleicht. Kepler und Galilei setzten Kopernikus durch, weil die zahlreichen Beobachtungen von Tycho Brahe, der im Ptolemäischen Weltbild verharrte, durch das Kopernikanische Weltbild einfacher beschrieben werden konnten als durch das Ptolemäische. Es ging also um die beste Beschreibung identischer Fakten.

Das Weltbild von Bofinger aber passt nur zum Zustand der Unterbeschäftigung, erklärt also längst nicht alle Fakten. Und das Weltbild der Vollbeschäftigung in der herkömmlichen Neoklassik passt gerade nicht zum Zustand der Unterbeschäftigung. Nur ein Weltbild, das sowohl Voll- als auch Unterbeschäftigung mit einbezieht, wird der Komplexität der modernen Welt gerecht - und das macht die neoklassische Synthese. Bofinger missversteht unsere Annahme der Vollbeschäftigung als eine Behauptung über die reale Welt. Er ignoriert das übliche analytische Verfahren des Gedankenexperiments: wie z. B. das der Fallgeschwindigkeit eines Körpers, wenn es keine Luftreibung gäbe. Also Wissenschaft wie vor Galilei?

Dabei blendet Bofinger das allgemeine Phänomen der Substitution in seinem Verständnis der Neoklassik völlig aus. Er stellt unsere Theorie so dar, als wenn die auf null sinkenden Zinsen keinen Handlungsbedarf auslösen; denn es werde ja Vollbeschäftigung bereits vorausgesetzt. (Bofinger, 2020, 578). Wir haben uns mit dieser Diskussion in Kapitel 2 unseres Buches befasst. Weiterhin bietet Weizsäcker (2019) eine Analyse der Wirkung der intertemporalen Substitution auf die Wohlfahrt der Bürger im Detail. Wir haben dort mit einem erheblichen theoretischen und empirischen Aufwand die intertemporalen Substitutionskräfte abgeschätzt. Ergebnis: Selbst bei Vollbeschäftigung würde die Abwesenheit von Staatsschulden den Wohlstand im Steady State um vielleicht $40 \%$ reduzieren, im Vergleich zum optimalen Zustand einer Staatsschuld, die zu einem risikofreien Realzins gleich der Wachstumsrate führt. Und wir fügen hinzu, dass bei diesem Wohlfahrtsverlust noch nicht mitgerechnet ist, dass die Wahrscheinlichkeit von hoher Beschäftigung in diesem Zustand ohne Staatschulden sehr viel kleiner ist als beim optimalen Stand der Staatsschulden.

\section{Substitution und Sparquote}

In ähnlicher Weise lässt sich der Begriff der impliziten Staatsschulden im Rahmen des Umlageverfahrens bei der gesetzlichen Rentenversicherung durch Substitution erklären. 
Er verweist darauf, dass die Nettosparquoten der privaten Haushalte in vielen Ländern zwischen 1980 und 2017 gefallen seien, was unserer These der wachsenden Zukunftsorientierung menschlichen Verhaltens widerspreche (Bofinger, 2020, 579). Aber er ignoriert unsere ausführlichen Darlegungen über den engen Substitutionszusammenhang zwischen dem „Zwangssparen“ in der gesetzlichen Rentenversicherung und dem freiwilligen Sparen. Dieser Zusammenhang ist für uns doch der Grund dafür, dass wir das Ergebnis des "Zwangssparens“ als privates Vermögen ansehen, dem beim Verpflichteten, dem Staat, eine entsprechende Verbindlichkeit gegenübersteht. In jedem von Bofinger aufgeführten Land ist die von uns definierte erweiterte Sparquote zwischen 1980 und 2015 gestiegen.

Wenn man das extremste Beispiel der gesunkenen eng definierten Sparquote, Italien, genauer ansieht, so erstaunt doch zugleich, dass das Medianvermögen (in der traditionellen Definition) der italienischen Haushalte über dem deutschen liegt. Offenbar hat sich dort auch ohne die Rentenansprüche der Sozialversicherung über die vergangenen Jahrzehnte viel Vermögen angesammelt. Der Grund ist der in Italien weit verbreitete Immobilienbesitz, der wegen gefallener Zinsen stark an Wert zugenommen hat. Diese Wertsteigerung ist nicht Teil der statistisch aufgeführten Sparquote. Diese Immobilien sind nach dem Beitritt zur Eurozone und damit erheblich niedrigeren Zinsen stark beliehen worden, was sich in einer niedriger ausgewiesenen Sparquote niederschlägt. All diese Substitutionsvorgänge werden in Bofingers Kritik des neoklassischen Ansatzes ignoriert.

Geht es dann um das nicht-neoklassische Gegenstück, das keynesianische Modell, kommt das Substitutionsphänomen plötzlich ins Blickfeld. Hier schreibt Bofinger: „Aus der Perspektive des keynesianischen Modells ist das Umlagesystem jeder Form der kapitalgedeckten Altersvorsorge überlegen. Es ermöglicht den Arbeitnehmern, in der Zukunft ein Einkommen zu sichern, ohne dass es dafür in der Gegenwart zu einem gesamtwirtschaftlichen Konsumverzicht kommen muss." (Bofinger, 2020, 580) Diese Aussage ist so apodiktisch formuliert, dass sie wohl auch bei einem Realzins von $10 \%$ p.a. gelten soll. Dies kann aber kaum so gemeint sein. Mehr noch: hier wird implizit die Substitution zwischen freiwilliger Ersparnis und gesetzlicher Altersvorsorge anerkannt. Denn die würde bei einem Kapitaldeckungssystem ja dadurch neutralisiert, dass die Sozialversicherung die geringe freiwillige Sparleistung durch institutionelle Sparleistung kompensiert. Das würde nach Bofinger den gesamtwirtschaftlichen Konsum konterkarieren, weil die Gesamtnachfrage entsprechend niedriger wäre. Dieses Denkgebäude geht allenfalls auf, wenn man anerkennt, dass es eine enge Substitution zwischen gesetzlicher Altersvorsorge und freiwilliger Sparleistung gibt. Warum wird derselbe Substitutionstatbestand beim keynesianischen Denkgebäude berücksichtigt, jedoch beim neoklassischen Denkgebäude ignoriert?

\section{Geld und die Steady-State-Analyse}

Eckhard Hein und Peter Bofinger werfen uns vor, Geld aus unserer Analyse ausgeklammert zu haben. Hierzu ist zunächst zu bemerken, dass in einem dynamischen makroökonomischen Modell natürlich Geld und Zentralbankgeld als separate Größe vorkommen müssen. Wir haben jedoch nicht das Ziel verfolgt, ein neues Modell zur makroökonomischen Dynamik zu entwickeln. Uns ging es in diesem Zusammenhang in erster Linie darum zu zeigen, welche Bedeutung Höhe und Form der Staatsschulden für eine adäquate dynamische Modellierung des gesamtwirtschaftlichen Geschehens besitzen. In der Moderne besteht Geld als Zahlungsmittel entweder aus Zentralbankgeld (Banknoten, Bankguthaben bei der Zentralbank) oder aus Giralgeld in Form von Guthaben der Nichtbanken bei den Banken. In unserem Buch sind beide Geldformen berücksichtigt, wenn auch - für die von uns vorgenommene Steady-State-Analyse - jeweils als Teile größerer Aggregate. Das Zentralbankgeld steht auf der Passivseite der Zentralbankbilanz. Die Zentralbank gehört dem Staat. Das Zentralbankgeld ist Teil der Staatsschulden und fließt damit in den Posten der Nettostaatsschulden ein.

Das private Vermögen der von uns betrachteten Volkswirtschaft ist die Aktivseite der konsolidierten Bilanz des privaten Sektors. Darin enthalten sind die Guthaben der privaten Wirtschaftssubjekte bei den Banken, insbesondere das Giralgeld. Es wird allerdings saldiert mit den Aktiva der Banken. Für eine Analyse der täglichen, wöchentlichen, monatlichen, vierteljährlichen Schwankungen des Wirtschaftsgeschehens ist es unabdingbar, dass der Geldbestand der Volkswirtschaft explizit dargestellt wird. Für unsere Steady-StateAnalyse ist dies nicht nötig. ${ }^{1}$ In dem kapitaltheoretisch fundierten „Metamodell“, das wir für die Steady-State-Analyse verwenden, kommt explizit kein Geld vor - so wie auch keine makroökonomische Produktionsfunktion oder eine explizit formulierte Nutzenfunktion des repräsentativen Haushalts vorkommt. Es fehlt auch eine Größe, die den Anteil des Staates oder seiner Steuereinnahmen am Sozialprodukt anzeigt. Der volkswirtschaftliche Gesamtkörper muss nur einer kleinen Zahl von Annahmen genügen. Dazu gehört z. B. die stetige Differenzierbarkeit von bestimmten Größen nach dem im jeweiligen Steady State vorherrschenden realen risikobereinigten Zinssatz. Es gehört dazu, dass sich der Fiskus am Kapitalmarkt finanzieren kann, wenn er genau diesen risikobereinigten Zinssatz zu zahlen bereit ist. Oder die Annahme,

1 Für eine Analyse langfristiger Wachstumsprozesse ist eine monetäre Analyse nicht zwangsläufig notwendig, insbesondere bei SteadyState-Analysen. So verfuhren sowohl die Klassiker von Adam Smith bis Karl Marx (Samuelson, 1974) als auch die einflussreichsten Ökonomen nach Keynes. 
dass dieser risikobereinigte reale Zinssatz ein (möglicherweise verzerrtes) Preissignal für die Grenzproduktivität des Kapitals darstellt. Letztere können wir ganz abstrakt erfolgreich definieren, wenn bestimmte Annahmen für das Metamodell gelten.

Natürlich ist die Annahme einer Solow-Produktionsfunktion immer bestenfalls eine Approximation der Realität. Gleiches gilt für die volkswirtschaftliche Geldmenge als Proxy für die Wirkung des Geldes auf das volkswirtschaftliche Geschehen. Da wir aber in unserem Metamodell weder die Solow-Approximation noch die genaue Wirkung des Geldes untersuchen, brauchen wir die Qualität dieser jeweiligen Approximationen auch nicht zu analysieren. Daher ist es nicht erforderlich, die Geldmenge explizit zu spezifizieren.

\section{Strom- und Bestandsgrößen}

Wir haben in unserem Buch aufgrund des Bezugs zur Kapitaltheorie mit volkswirtschaftlichen Bestandsgrößen operiert. Bestandsgrößen wie Vermögen und Kapitalstock stehen in einem klar definierten Zusammenhang mit Stromgrößen wie Sparen und Investieren. Immer mehr moderne makroökonomische Modelle verwenden sowohl Strom- als auch Bestandsgrößen und achten auf eine entsprechend konsistente Beziehung zwischen diesen Größen (StockFlow Consistent Models). Eckhard Hein ist uneingeschränkt zuzustimmen, wenn er auf die generelle Notwendigkeit hinweist, dass ökonomische Modelle „grundlegende makroökonomische und finanzielle Strom- und Bestandsrechnungsbeziehungen“ (Hein, 2020, 585) beachten müssen. Dieser Anforderung entsprechen wir in den empirischen Berechnungen, für die wir Vermögensbilanzen für verschiedene Wirtschaftssektoren wie Unternehmen, Privathaushalte und den Staat erstellen und dabei auf Daten aus den Volkswirtschaftlichen Gesamtrechnungen zurückgreifen. Wir haben außerdem in Kapitel 7 gezeigt, dass unser auf Bestandsgrößen basierendes Erklärungsmodell äquivalent zu einer Modellierung mit Stromgrößen ist. Damit und generell mit unserem neuen Denkansatz konnten wir zeigen, dass in einer Gesellschaft, die von zunehmender durchschnittlicher Lebenserwartung der Bürger sowie vom Erreichen der Grenze für die Mehrergiebigkeit längerer Produktionsumwege geprägt ist und in der eine zu geringe Staatsschuld be- steht, ein „Bestands- und Stromungleichgewicht“ (Spahn, im Erscheinen) herrscht. Dessen negative ökonomische Auswirkungen können im Interesse der Vollbeschäftigung und zur Vermeidung von Inflation und dauerhaften Niedrigzinsen nur durch eine signifikante Ausweitung der Staatsschulden bekämpft werden.

\section{Literatur}

Arnott, R. J. und J. E. Stiglitz (1979), Aggregate Land Rents, Expenditure on Public Goods, and Optimal City Size, Quarterly Journal of Economics, 93(4), 471-500.

Bofinger, P. (2020), Sparen und Investieren im Spannungsfeld widerstreitender Paradigmen, Wirtschaftsdienst, 100(8), 577-581, https://www. wirtschaftsdienst.eu/inhalt/jahr/2020/heft/8/beitrag/sparen-undinvestieren-im-spannungsfeld-widerstreitender-paradigmen.html (11. September 2020).

Haug, T. (2018), Berechnung der Pensions- und Rentenanwartschaften in den volkswirtschaftlichen Gesamtrechnungen. Berechnungsmethodik und Ergebnisse, Wirtschaft und Statistik, 2, 77-90.

Hein, E. (2020), Sparen und Investieren im 21. Jahrhundert - die postkeynesianische Perspektive, Wirtschaftsdienst, 100(8), 582-585, https://www.wirtschaftsdienst.eu/inhalt/jahr/2020/heft/8/beitrag/ sparen-und-investieren-im-21-jahrhundert-die-post-keynesianischeperspektive.html (11. September 2020).

Keynes, J. M. (1936), The General Theory of Employment, Interest and Money, Macmillan.

Lequiller, F. und D. Blades (2014), Understanding National Accounts, 2. Auflage, OECD Publishing.

Piketty, T. (2014), Das Kapital im 21. Jahrhundert, C. H. Beck.

Samuelson, P. A. (1948), Economics. An Introductory Analysis, McGrawHill.

Samuelson, P. A. (1974), Marx as a Mathematical economist, in G. Horwich und P. A. Samuelson (Hrsg.) Trade, Stability and Macroeconomics. Essays in Honor of Lloyd A. Metzler, 269-307, Academic Press.

Sinn, H.-W. (2020), Staatsverschuldung und dynamische Ineffizienz: Warum der Münchhausen-Trick nicht funktioniert, Wirtschaftsdienst, 100(8), 572-577, https://www.wirtschaftsdienst.eu/inhalt/jahr/2020/ heft $/ 8 /$ beitrag/staatsverschuldung-und-dynamische-ineffizienz-warum-der-muenchhausen-trick-nicht-funktioniert.html (11. September 2020).

Spahn, P. (2021), Bestands- und Stromungleichgewicht in der Aging Economy - Bemerkungen zu Sparen und Investieren im 21. Jahrhundert, in H. Krämer und J. Schmidt (Hrsg.), Wirtschaftspolitische Beratung in der Krise, Metropolis-Verlag (im Erscheinen).

Weizsäcker, C. C. von (2019), Capital Theory of the Steady State - Or: T = Z - D, https://www.coll.mpg.de/Weizsaecker/CapitalTheory2019 und https://www.springer.com/9783658273620 (3. September 2020).

Weizsäcker, C. C. von und H. Krämer (2020), Sparen und Investieren im 21. Jahrhundert: Das Ende der Kapitalknappheit, Wirtschaftsdienst, 100(8), 569-572, https://www.wirtschaftsdienst.eu/inhalt/jahr/2020/ heft/8/beitrag/sparen-und-investieren-im-21-jahrhundert-das-endeder-kapitalknappheit.html (11. September 2020).

Weizsäcker, C. C. von und H. Krämer (2019), Sparen und Investieren im 21. Jahrhundert - Die große Divergenz, Springer Gabler.

Title: On the Relationship Between Interest Rates and Economic Growth: Theory and Empirical Evidence

Abstract: In the August 2020 issue of Wirtschaftsdienst Hans-Werner Sinn, Peter Bofinger and Eckhard Hein made critical comments on our book "Sparen und Investieren im 21. Jahrhundert - Die große Divergenz" (Saving and Investing in the 21st Century - The Great Divergence). In this article, we respond to their critique. In particular, we deal with the controversial phenomenon of dynamic inefficiency, with the criticisms of our concept of implicit public debt, the interpretation of economic paradigms, and the allegation of having neglected monetary aspects in our theory.

JEL Classification: E01, E22, D81 